\title{
Using Nonlinear Dynamics for Signat Analysis in Transpalpebral Rheoophthalmography
}

DOI: $10.17691 /$ stm2018.10.3.20

Received December 16, 2017

P.V. Luzhnov, PhD, Associate Professor, Department of Medical and Technical Information Technologies";

D.M. Shamaev, Assistant, Department of Medical and Technical Information Technologies';

A.A. Kiseleva, Student, Department of Medical and Technical Information Technologies';

E.N. Tomdina, DSc, Professor, Chief Researcher, Department of Refraction Disorders, Binocular Vision

and Ophthalmoergonomics':

D.D. Khoziev, PhD Student;

O.A. Kiseleva, MD, DSc, Head of the Department of Glaucoma²

1Bauman Moscow State Technical University, 5/1, $2^{\text {nd }}$ Baumanskay St., Moscow, 105005, Russia;

2Moscow Helmholtz Research Institute of Eye Diseases, 14/19 Sadovaya-Chernogryazskaya St., Moscow, 105062, Russia

The aim of the study was to analyze signals of transpalpebral rheoophthalmography (TP ROG) by using methods of nonlinear dynamics, to characterize the parameters of nonlinear filtration, and to verify these parameters by testing the TP ROG signals in patients with primary open-angle glaucoma (POAG).

Materials and Methods. Parameters of the nonlinear filter were determined from the data reported by others and from our own studies on multiple TP ROG signals recorded in 10 subjects (mean age $54.2 \pm 15.4$ years) with no ophthalmic abnormalities. Adjustment and verification of these parameters were performed using TP ROG signals from 10 patients with POAG, divided into two groups: group 1 - six patients with stage II POAG (mean age $72.0 \pm 8.2$ years) and group 2 - four patients with stage III POAG (mean age $69.4 \pm 6.8$ years).

To analyze the obtained TP ROG signals, a method of nonlinear dynamics with the signal attractor formation was employed; within this approach, the time delay value was selected, the phase space dimension was determined, and the attractor was created within the selected coordinates. After calculating the barycenter of each attractor, the TP ROG signals were graphically analyzed in the phase space of the attractors representation.

Results. Using the nonlinear dynamics to process TP ROG signals provided for a better signal differentiation by time as compared with the previously used signal processing. The nonlinear filtering makes it possible to represent the TP ROG signal (within the selected coordinates) by an attractor, which characterizes the amplitude, temporal and structural features of the signal directly related to the biophysical parameters of eye blood vessels. The technique of signal analysis was also tested in patients with POAG. It was found that the barycenter of the newly created attractor can move within the selected coordinates, and this change depends on the stage of the disease. The results allow one to help diagnose the disease in early stages; the method can also be used to look further into the relationship between eye hemodynamics and glaucoma.

Conclusion. A technique is proposed for a quantitative comparison between different TP ROG signals; the method is based on the location of the attractor barycentre within the selected coordinates. The suggested nonlinear filtering algorithm makes it possible to discern between those signals considering the totality of its amplitude and temporal characteristics. The technique is verified by testing TP ROG signals from patients with POAG of different stages, thus further supporting this method of processing and analyzing information on the eye blood flow.

Key words: rheoophthalmography; transpalpebral rheoophthalmography; nonlinear dynamics; nonlinear filtering; eye blood flow diagnosis; glaucoma.

\section{Introduction}

At present, there is a growing interest in studying the time series of diagnostic data by methods of nonlinear dynamics [1, 2]. It is accepted that indices of various body functions can be seen as manifestations of the deterministic chaotic processes. In this respect, the methods of nonlinear dynamics can be helpful in obtaining new information about biological systems, for example, the circulatory system of human organs or tissues [3].

The normal vision, like any other body function, depends on the adequate trophic supply of the eye tissues, in particular by the adequate blood flow. In 
such eye disorders as myopia, diabetic retinopathy, glaucoma, retinal vascular occlusion, and others, hemodynamic measurements in the eye provide the ophthalmologist with additional information on the pathogenesis and give the doctor more instruments for early diagnosis and treatment. The high prevalence of the above illnesses necessitates further research into this field. Thus, among the causes of visionassociated disability in Russia, glaucoma takes the $1^{\text {st }}-2^{\text {nd }}$ place, and complicated myopia - the $3^{\text {rd }}$ (in the children's disability - the $2^{\text {nd }}$ ). In this regard, diagnostic examination of the intraocular blood flow may find its use in early diagnosis, disease monitoring and assessment of treatment efficacy.

Rheoophthalmography is a method for determining the eye blood flow. Its principle is based on measuring the tissue resistance (impedance) as probed by a high-frequency lowamplitude current passing through the eye tissues. The classical technique of rheoophthalmography requires electrodes (for example, those proposed by Chibirene) to be installed in the perilimbal area of the eye [4]. This manipulation necessitates the conjunctiva to be anesthetized, which increases the risk of infection or eyeball damage.

In reports [5-7], an original technique of transpalpebral rheoophthalmography (TP ROG) is described; there, electrodes are placed on the closed eyelid [8]. In the process of developing this technique, it incorporated the results of mathematical modeling of the eyeball vascular bed anatomy, which allowed the authors to increase the accuracy of the measurements $[9,10]$.

The aim of the study was to analyze TP ROG signals using the methods of nonlinear dynamics, to characterize the parameters of nonlinear filtration, and to verify these parameters by testing the proposed technique in patients with primary open-angle glaucoma.

\section{Materials and Methods}

For TP ROG signals, as for any other rheographic signals, the rheogram analysis includes both the qualitative and quantitative components [11]. An important task of the qualitative analysis is to characterize the pulse wave of the rheogram [12]. However, the existing approach to analyzing the pulse wave in TP ROG signals is not objectified.

It is known that changes in the blood flow detected with the rheographic signals reflect biophysical, biomechanical and hydrodynamic changes in the tone and innervation of blood vessels. The structure of the rheographic signal and its hemodynamic interpretation are described, for example, in [11, 13] (Figure 1).

In the rheographic signal (Figure 1 (b)), a cardiointerval begins with a steep ascent, which reaches

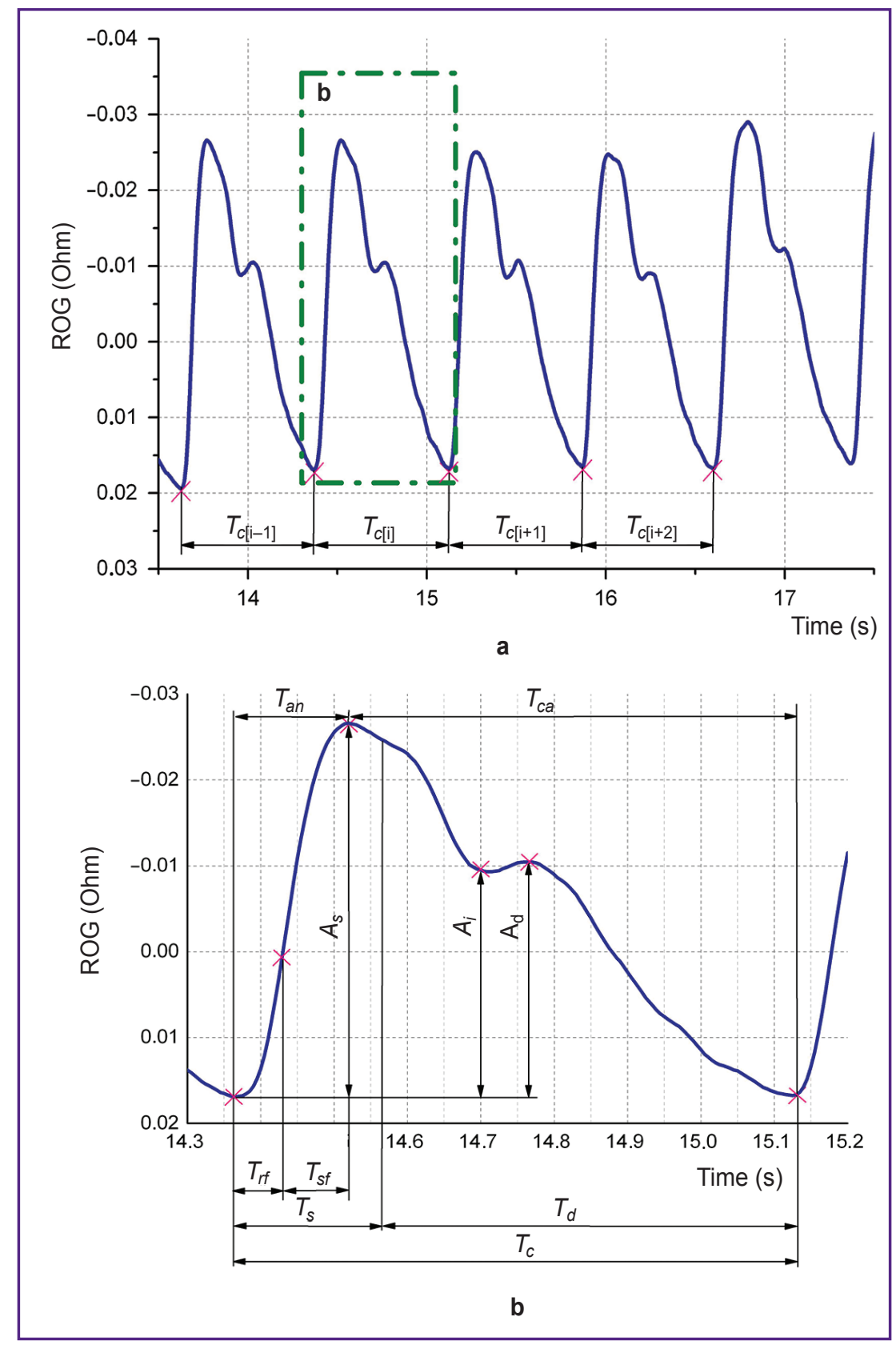

Figure 1. Structure of a rheographic signal:

(a) a rheoophthalmographic signal with accentuated cardiointervals; (b) the major temporal and amplitude parameters of a rheographic signal; $T_{s}$ - duration of the systole; $T_{d}$ - duration of the diastole; $T_{c}$ - duration of the cardiointerval 
the apex of the systolic wave $\left(A_{s}\right)$. After that, the signal descends until the next cardiointerval begins. The ascending part of the signal is called anacrotic $\left(T_{a n}\right)$, the entire descending part of the signal is called catacrotic $\left(T_{c a}\right)$. The steepness of the anacrotic part is not constant over the signal ascent; the anacrotic signal includes the rapid blood filling phase $\left(T_{\text {rf }}\right)$ and the slow filling phase $\left(T_{\text {sf }}\right)$ [11]. The systolic apex determines the amplitude of the entire rheographic signal and forms a systolic wave. The systolic wave is followed by a local minimum signal, called an incisure $\left(A_{i}\right)$. After the incisure, a normal diastolic wave $\left(A_{d}\right)$ is recorded.

The amplitude and shape of the rheographic signal are influenced by the nature of the blood flow and the elastic-viscous properties of blood vessel walls in both the nearby and remote vessel segments. The principle hemodynamic mechanism behind the anacrotic and catacrotic parts is the different rates of blood inflow and outflow in the area of interest. In the anacrotic interval, the volume velocity of blood filling exceeds that of the outflow. During the catacrotic interval, the blood volume leaving the area is greater than the incoming blood volume. The rapid blood filling interval is thought to reflect, firstly, the physiological heterogeneity of the left ventricular ejection process, when up to $80 \%$ of the heart blood is ejected into the blood stream (the rapid ejection phase) and, secondly, the hemodynamic characteristics of large arteries. The slow blood filling period reflects the second (reduced) phase of left ventricle ejection. The catacrotic and diastolic wave are determined by the rate of blood flow through arterioles and pre-capillaries into the capillary system, as well as the elastic-viscous properties of the distal blood vessels $[11,13]$.

Together, all these factors influence the form of the pulse wave and determine the qualitative analysis result of the cardiointerval on the rheographic signal. To create an algorithm for analyzing the TP ROG signals, we proposed to employ the tools of nonlinear dynamics; such an algorithm would incorporate both the amplitude and pulse wave characteristics of TP ROG signals. In studies on various systems, including biological ones, the use of nonlinear dynamics involves nonlinear models and filters. As a rule, these are represented by differential equations and discrete mappings [14]. The methods of nonlinear dynamics are based on creating an attractor over a series of time points. In our study, the attractors represented by the time-sampled signal produced by TP ROG. This series is analyzed using the method of coordinate delay, i.e. a sequence of vectors is created in the phase space [15]; these vectors are obtained from the elements of the time series with an interval determined by the time delay parameters.

To determine the nonlinear filtering parameters, it is necessary to select the time delay and the dimension of the phase space. Based on the available information on the factors that influence the signal structure (see Figure 1), we selected the phase of rapid blood filling and the existence of a systolic and a diastolic wave. Thus, the dimension of the phase space for the filter being developed must be at least three. Therefore in this presentation, after the nonlinear filtering the result is shown within the three-dimensional coordinate space (X1, X2, and X3).

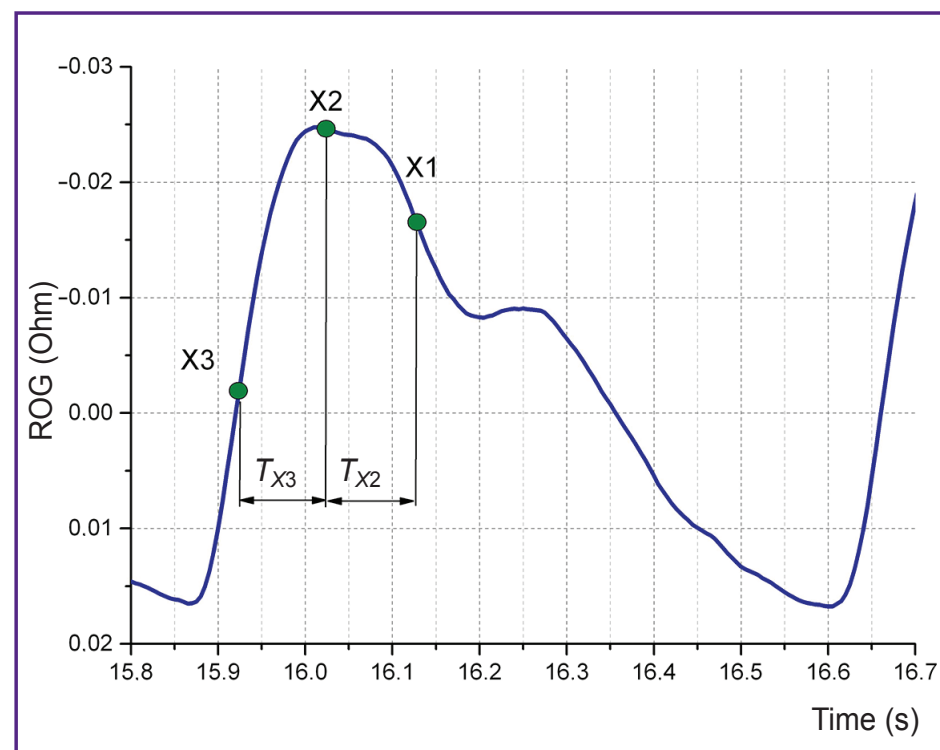

a

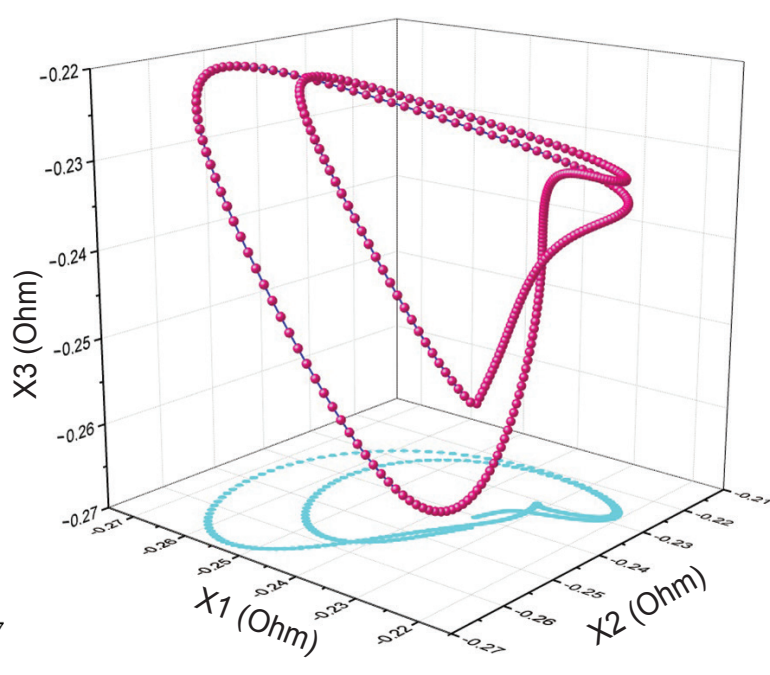

b

Figure 2. Creation of a signal attractor in TP ROG:

(a) determining the three-dimensional coordinates; (b) creating an attractor for a TP ROG signal from a subject without ophthalmic disorders 
With a nonlinear filter, the time delay parameter depends on the sampling frequency and it correlates with the phase duration ratio of the signal. In addition, this is determined by the heart rate of the patient at the time of signal recording, i.e. by the duration of the cardiointerval and the pulse wave. All signals used in this study were recorded at a sampling rate of $200 \mathrm{~Hz}$. For a heart rate of 60 per minute, one cardiointerval is probed 200 times (i.e. measurements are made). In our calculations, this number represented the cumulative duration of all phases of the pulse wave.

Initially, the time delay was chosen so that the correlation between the neighboring signal samples was minimal. This requirement was met with a delay value of 100 samples, i.e. a half the cardiointerval duration in the original signal. Assuming each of the two time delays of the selected phase space equal to each other, we get $T_{X 2}=50$ samples and $T_{X 3}=50$ samples. An example of a created signal attractor for one subject is shown in Figure 2.

To record TP ROG signals we used an impedance sensitive transducer with original software and a tetrapolar lead system [16]. During the examination, the patient was resting in a horizontal position with his/her eyes closed; the lead system was placed on the eye and fixed with a knitted helmet. The study was conducted at the Department of Glaucoma of the Moscow Helmholtz Research Institute of Eye Diseases. We recorded and analyzed TP ROG signals of 10 subjects (mean age $54.2 \pm 15.4$ years) without ophthalmic abnormalities, as well as 10 patients with primary open-angle glaucoma (POAG); the latter were divided into two groups: group 1 - six patients (mean age $72.0 \pm 8.2$ years) with stage II POAG, and group 2 - four patients (mean age $69.4 \pm 6.8$ years) with stage III POAG.

The study was conducted in accordance with the Helsinki Declaration (2013) and approved by the Ethics Committee of the Moscow Helmholtz Research Institute of Eye Diseases. An informed consent was obtained from each patient.

\section{Results}

Using the shape of the attractor in the given phase space we calculated the position of its mass center or the barycenter. By comparing positions of all mass centers in the group, which varied between the subjects, we obtained the occurrence rate for this attractor; the distance between the mass centers of the attractors was also calculated.

At the first stage, the TP ROG signals were analyzed for 10 subjects with no ophthalmic disorders. At $T_{X 2}=50$ samples and $T_{X 3}=50$ samples, the standard deviation (reflecting the spread of the attractors mass centers within the group), was high; due to that, we needed to optimize the values of $T_{X 2}$ and $T_{X 3}$ in the nonlinear filter. Taking into account the systole durations and the period of rapid blood filling, we selected the ratio of $T_{X 2} /$ $T_{X 3}=2 / 5$. For the time delay parameters $T_{X 2}$ and $T_{X 3}$, the ratios of 20/50, 16/40, $8 / 20$ were tested. The quantitative assessment of the attractor mass centers variations for the above ratios is shown in Figure 3. Upon the data analysis, the smallest data variation was found at $T_{X 2} /$ $T_{X 3}=16 / 40$.

Thus, based on these results (see Figure 3), the following values were chosen for the nonlinear signal filtering: $T_{X 2}=16$ samples, $T_{X 3}=40$ samples.

Using the obtained parameters of the nonlinear filter, we created attractors for TP ROG signals obtained from subjects without ophthalmic problems. An example of such an attractor and its projections on the plane of the phase space is shown in Figure 4.

In order to simplify the calculation of the mass center location, it was conditionally approximated by a figure formed by two pyramids with a common quadrangular base. The vertices of the pyramids were determined by the maximum and minimum values of each of the three axes of the phase space. The lateral projections of the pyramids are triangles, and the projection of the base is a quadrangle (the projections are indicated by the red lines in Figure 4). Then, it becomes possible
Figure 3. Relative change in the standard deviation of the attractor mass center position at different time delay ratios

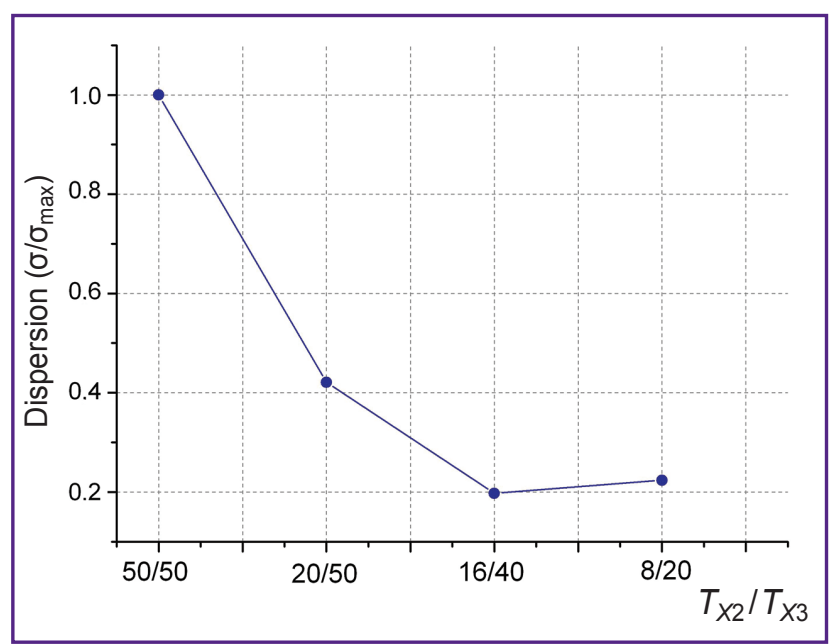




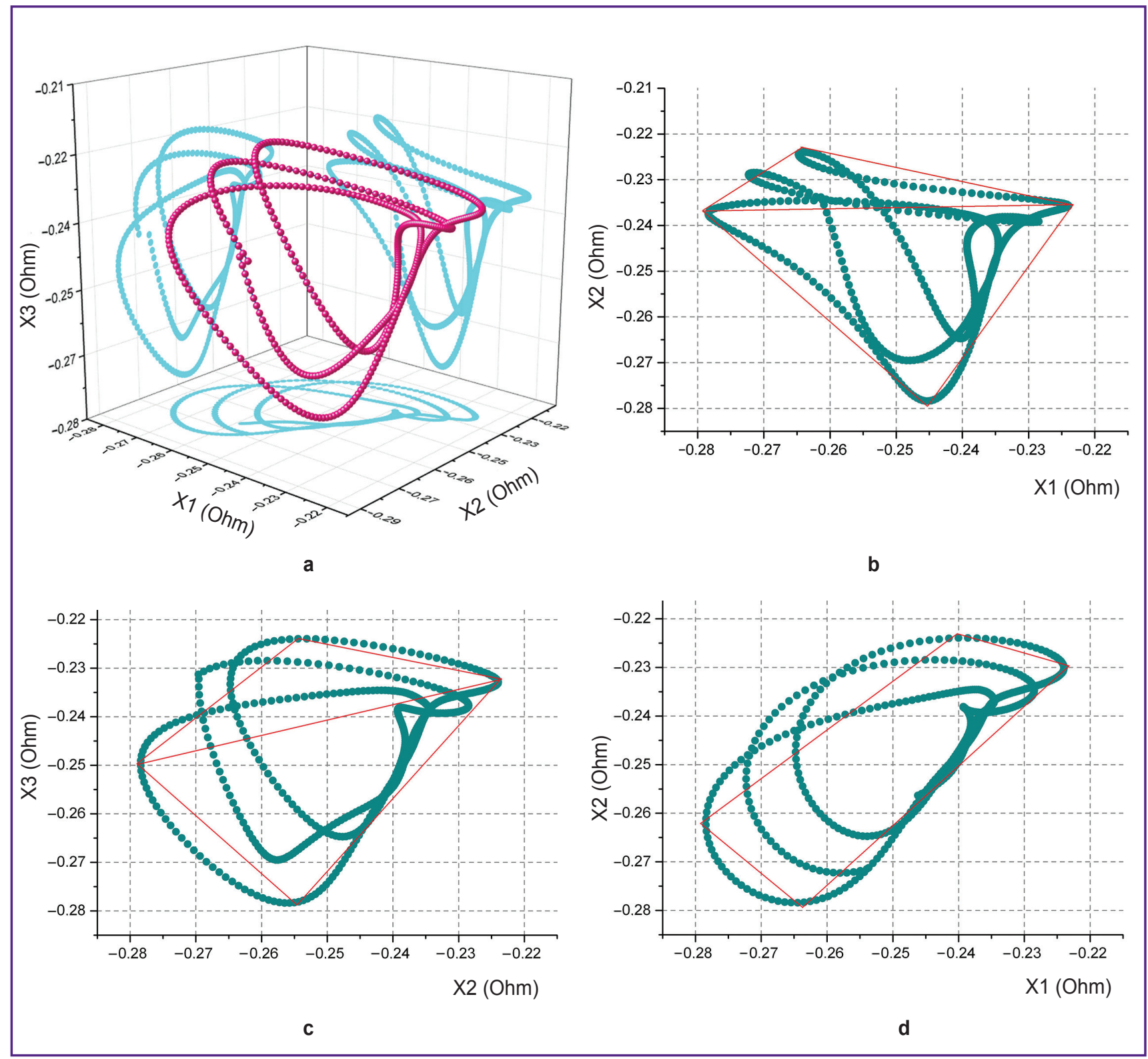

Figure 4. An attractor (a) and its projections (b)-(d) for a TP ROG signal from a subject without ophthalmic disorders

to determine the attractor mass center and the mass centers of the two pyramids - using the triangular projections and making the calculation for the mass center within the triangle frame.

Verification of the proposed nonlinear filtering technique was performed using TP ROG signals from patients with POAG. The signals from 10 patients (10 eyes) with non-operated POAG at stages II and III were analyzed.

An example of the TP ROG signal attractor for a patient with POAG is shown in Figure 5.

As can be seen from Figure 4 and 5, the shape and position of the attractor, approximated by two triangles on the lateral projections are apparently different from those in control subjects. Based on this relationship, we can propose a new condition to characterize the existence and stage of POAG; and that is the attractor barycenter position. By calculating the barycenter of each attractor, it becomes possible to graphically analyze TP ROG signals on the representation plane of the attractors.

\section{Conclusion}

Here we describe the technique for a quantitative comparison of signals recorded via TP ROG based on the attractor barycentre position in the space of selected coordinates. The proposed nonlinear filtering algorithm allowed us to discern between the signals by the totality of their amplitude and temporal characteristics. 


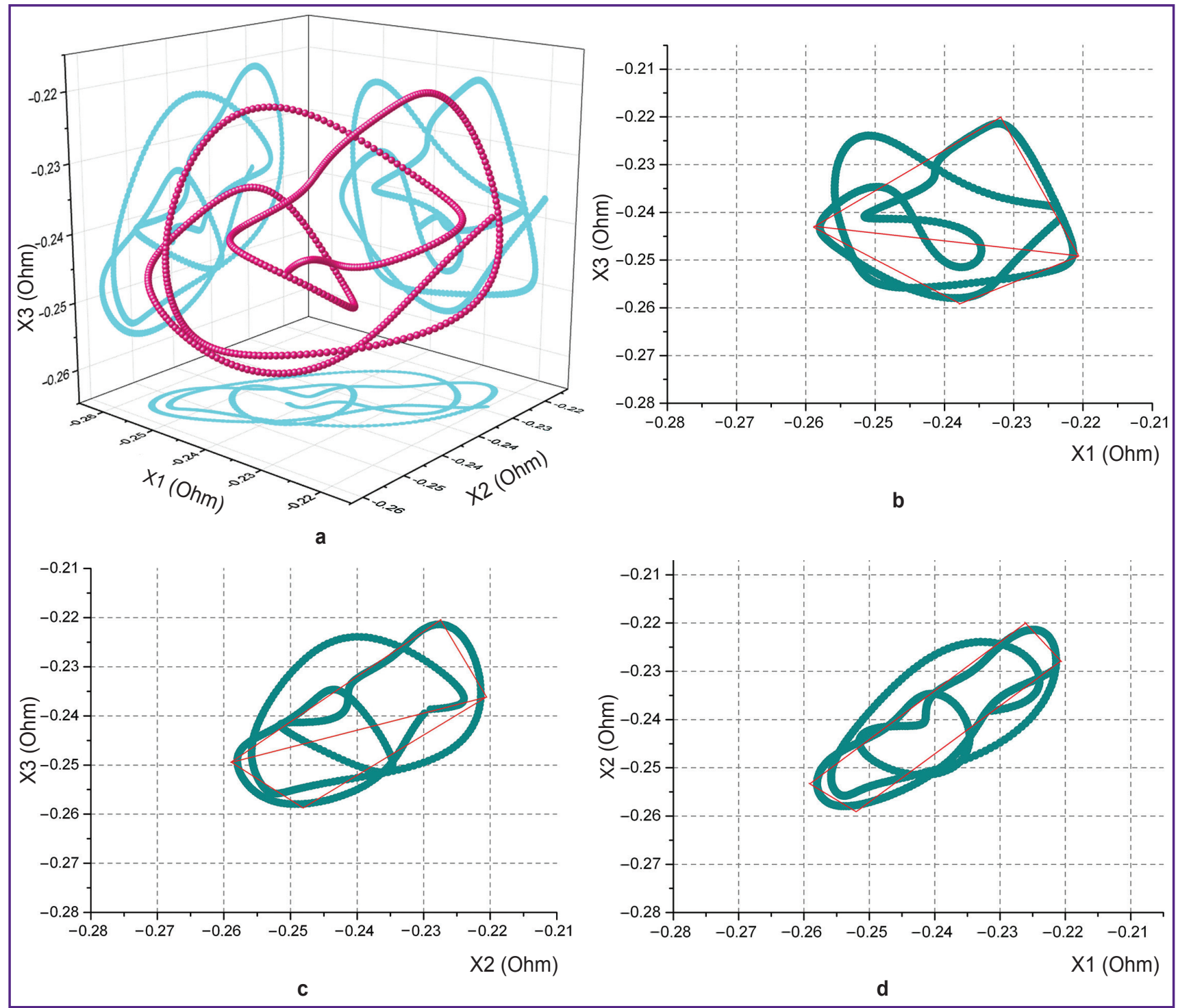

Figure 5. An attractor (a) and its projections (b)-(d) for a TP ROG signal from a patient with primary open-angle stage II glaucoma

The method has been verified by using the signals from patients with POAG, which further supports its applicability for processing and analyzing diagnostic information on disorders of eye blood flow.

Financial support. The work was supported by grant No.18-08-01192 from the Russian Foundation for Basic Research.

Conflict of interest. The authors declare they have no conflict of interest.

\section{References}

1. Goldberger A.L. Non-linear dynamics for clinicians: chaos theory, fractals, and complexity at the bedside. Lancet 1996; 347(9011): 1312-1314, https://doi.org/10.1016/s01406736(96)90948-4.

2. Subramaniam B., Khabbaz K.R., Heldt T., Lerner A.B.,
Mittleman M.A., Davis R.B., Goldberger A.L., Costa M.D. Blood pressure variability: can nonlinear dynamics enhance risk assessment during cardiovascular surgery? J Cardiothorac Vasc Anesth 2014; 28(2): 392-397, https://doi.org/10.1053/j. jvca.2013.11.014.

3. Ishbulatov Y.M., Karavaev A.S., Ponomarenko V.I., Kiselev A.R., Sergeev S.A., Seleznev E.P., Bezruchko B.P., Prokhorov M.D. Phase synchronization of elements of autonomic control in mathematical model of cardiovascular system. Nelineinaya dinamika 2017; 3(13): 381-397, https://doi. org/10.20537/nd1703006.

4. Lazarenko V.I. Funktsionalnaya reografiya glaz [Functional rheography of the eyes]. Krasnoyarsk: Rastr; 2000; $160 \mathrm{p}$.

5. Luzhnov P.V., Parashin V.B., Shamaev D.M., lomdina E.N., Markosyan G.A., Napylova O.A. Use of tetrapolar methods during rheoophtalmography for an estimation of blood supply inside an eye. Biomeditsinskaya radioelektronika 2012; 10: 18-21. 
6. Luzhnov P.V., Shamaev D.M., lomdina E.N., Tarutta E.P., Markosyan G.A., Shamkina L.A., Sianosyan A.A. Transpalpebral tetrapolar reoophtalmography in the assessment of parameters of the eye blood circulatory system. Vestnik Rossiiskoi akademii meditsinskikh nauk 2015; 70(3): 372-377, https://doi.org/10.15690/vramn.v70i3.1336.

7. Iomdina E.N., Luzhnov P.V., Shamaev D.M., Tarutta E.P., Kiseleva T.N., Markossian G.A., Ramazanova K.A., Napylova O.A., Kuryleva I.M., Shamkina L.A. An evaluation of transpalpebral rheoophthalmography as a new method of studying the blood supply to the eye in myopia. Rossijskij oftal'mologiceskij zurnal 2014; 7(4): 20-24.

8. Shamaev D., Luzhnov P., Pika T., lomdina E., Kleyman A., Sianosyan A. Applying transpalpebral rheoophthalmography to monitor effectiveness of the treatment of patients with glaucoma. International Journal of Biomedicine 2016; 6(4): 287-289, https://doi.org/10.21103/article6(4)_oa8.

9. Luzhnov P.V., Parashin V.B., Shamaev D.M., Shamkina L.A., lomdina E.N., Markosyan G.A., Napylova O.A., Tarutta E.P. Modelling features of various eye environments at rheoophthalmography. Biomeditsinskaya radioelektronika 2013; 10: 35-39.

10. Shamaev D.M., Luzhnov P.V., lomdina E.N. Modeling of ocular and eyelid pulse blood filling in diagnosing using transpalpebral rheoophthalmography. EMBEC \& NBC 2017; 65: 1000-1003, https://doi.org/10.1007/978-981-10-5122-7_250.
11. Ivanov L.B., Makarov V.A. Lektsii po klinicheskoy reografii [Lectures on clinical rheography]. Moscow: Antidor; 2000; $320 \mathrm{p}$.

12. Luzhnov P.V., Pika T.O., Shamaev D.M., Nikolaev A.P. Features of the form parameters definition during the rheographic signals analysis. Biomeditsinskaya radioelektronika 2015; 7: 25-30.

13. Sokolova I.V., Ronkin M.A., Maksimenko I.M. Osnovy pulsovoy gemodinamiki [Fundamentals of pulse hemodynamics]. Moscow: Samshit-izdat; 2007; 172 p.

14. Shilnikov L.P., Shilnikov A.L., Turaev D.V., Chua L. Metody kachestvennoy teorii $v$ nelineynoy dinamike [Methods of qualitative theory in nonlinear dynamics]. Moscow-Izhevsk; 2003; $428 p$

15. Gracia J., Seppä V.-P., Pelkonen A., KotaniemiSyrjänen A., Mäkelä M., Malmberg P., Viik J. Nonlinear local projection filter for impedance pneumography. EMBEC \& NBC 2017; 65: 306-309, https://doi.org/10.1007/978-981-10-51227 777.

16. Luzhnov P.V., Shamaev D.M., Iomdina E.N., Markosyan G.A., Tarutta E.P., Sianosyan A.A. Using quantitative parameters of ocular blood filling with transpalpebral rheoophthalmography. In: Eskola $\mathrm{H}$., Väisänen O., Viik J., Hyttinen J. (editors). EMBEC \& NBC 2017. IFMBE Proceedings, Vol. 65; p. 37-40, https://doi. org/10.1007/978-981-10-5122-7_10. 